\title{
Wie viel ist Ihre Arbeit wert?
}

\author{
Chefredakteur Dr. med. Dirk Einecke
}

\section{NACH DER ERSTEN OSTEOPOROSEFRAKTUR}

\section{Männerknochen besonders gefährdet}

Eine erste osteoporosebedingte Fraktur ist immer ein Alarmsignal - dafür dass bald eine weitere Fraktur folgen könnte. Dieser Zusammenhang ist für ältere Frauen gut dokumentiert - bei Männern fällt der Risikoanstieg aber noch dramatischer aus. Nach einer ersten Fraktur infolge eines geringen Traumas erleiden Männer 3,5-mal häufiger einen weiteren derartigen Knochenbruch (Frauen 2-mal häufiger), besonders gefährlich ist das erste Jahr nach einer Fraktur. Ihr Risiko entspricht damit dem eines 20 Jahre älteren Mannes ohne Fraktur. Zu diesem Ergebnis kommen australische Forscher, die die über einen Zeitraum von 15 Jahren gesammelten Daten von rund 4000 über 60-Jährigen ausgewertet haben. Eine Teilerklärung für die besonders zerbrechlichen Männerknochen: Eine Osteoporose wurde nur bei knapp $4 \%$ der betroffenen Männer medikamentös behandelt, bei den Frauen erhielten allerdings auch weniger als $25 \%$ eine notwendige Therapie.

JAMA 2007;297:387-94

\section{NACH VENÖSER THROMBOEMBOLIE}

\section{Kein besserer Schutz durch verlängerte Antikoagulation}

Nach einer ersten tiefen Venenthrombose (TVT) oder Lungenembolie (LE), die weder eine idiopathische Genese haben noch auf eine Thrombophilie zurückzuführen sind, wird heute eine orale Antikoagulation für die Dauer von drei Monaten empfohlen. Die Sekundärprophylaxe auf einen längeren Zeitraum auszudehnen, bringt wahrscheinlich keinen Nutzen, wie eine britische Studie zeigt. Rund 750 Patienten mit erstmaliger TVT oder LE hatten entweder drei oder sechs Monate lang Warfarin geschluckt. In beiden
Gruppen kam es bei 8\% der Patienten zu einer erneuten TVT oder LE, davon bei zwei Patienten in der 3-Monats-Gruppe und drei in der 6-Monats-Gruppe mit tödlichem Ausgang. Unter der längeren Antikoagulation wurden außerdem signifikant mehr schwere Blutungen beobachtet. Wenn man alle unerwünschten Ereignisse zusammen berücksichtigte, gab es keinen signifikanten Unterschied zwischen der üblichen und der verlängerten Behandlung ( $8 \%$ vs. $9 \%, p=0,79$ ).

BMJ; online 8. Februar 2007
Das „British Medical Journal“ geht in seiner aktuellen Ausgabe der Frage nach, ob britische Ärzte überbezahlt sind. Der Vergleich mit anderen $\mathrm{Na}$ tionen zeigt, dass dies zumindest für deutsche Ärzte nicht zutrifft. Niedergelassene Allgemeinärzte verdienen hier zwar mehr als in Italien oder Frankreich, aber deutlich weniger als britische oder US-amerikanische Kollegen (Abb.). Noch schlechter schneiden im Ländervergleich die deutschen Krankenhausärzte $a b$.

\section{Jahreseinkommen von niedergelassenen Allgemeinärzten (2003/2004)}

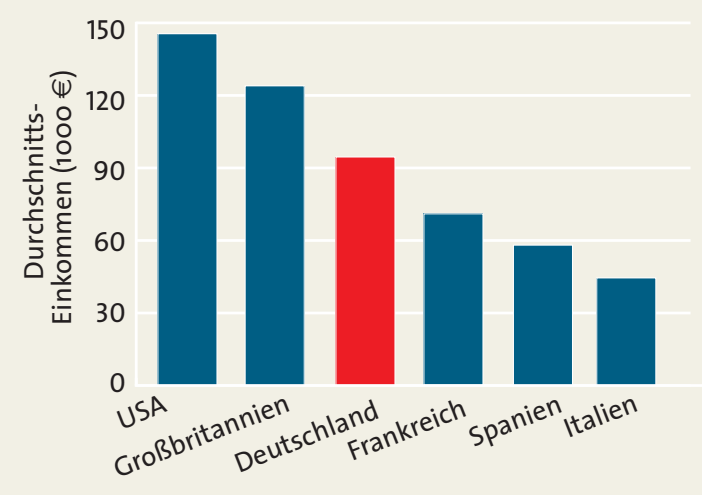

\section{ALZHEIMER-RISIKO ERHÖHT}

\section{Einsamkeit schadet dem Gehirn}

Ältere Menschen, die unter Einsamkeit leiden, haben ein doppelt so hohes Alzheimer-Risiko wie solche, die sich nicht allein fühlen. Wie eine Studie mit 823 älteren Personen ergab, ist dabei besonders die gefühlte Einsamkeit, weniger die tatsächliche soziale Integration von Bedeutung. Das Risiko war umso höher, je stärker das Gefühl der Verlassenheit war. Arch Gen Psychiatry 2007;64:234-40

\section{HOCHDRUCK IN DER SCHWANGERSCHAFT}

\section{KHK nach der Menopause}

Ein Schwangerschaftshochdruck ist nicht nur ungünstig für den Verlauf der Schwangerschaft, er bedeutet für die betroffenen Frauen auch ein erhöhtes Risiko, im späteren Leben an einer KHK zu erkranken. Dieser Zusammenhang stellte sich bei der Untersuchung von 491 postmenopausalen Frauen heraus, von denen $31 \%$ anamnestisch angaben, dass bei innen in der Schwangerschaft ein Hochdruck festgestellt worden war. Diese Frauen hatten ein um $57 \%$ erhöhtes Risiko für eine KHK als Frauen, die als Schwangere Jahrzehnte früher normale Blutdruckwerte aufgewiesen hatten. Hypertension; online 5. Februar 2007

\section{RISIKOFAKTOR BEWEGUNGSARMUT}

\section{Holt die Kinder von der Glotze!}

Bewegungsarmut erhöht bei Jugendlichen das Hypertonierisiko, unabhängig davon, ob sie übergewichtig sind oder nicht. Im Rahmen des National Health and Nutrition Examination Survey wurden 4500 Jugendliche im Hinblick auf Körpergewicht und körperliche Aktivität befragt. Man fand, dass 12- bis 15-Jährige, die besonders lange Zeit vor Fernseher oder Videogerät verbrachten, höhere Blutdruckwerte hatten als Altersgenossen, die sich mehr bewegten. J Adolesc Health 2007;40:166-72 